\title{
Validade das medidas referidas da massa corporal e estatura em universitários
}

\author{
Validation of self-reported measures of body mass and stature in college students
}

Thiago Ferreira de Sousa ${ }^{1}$, Aline Rodrigues Barbosa ${ }^{2}$

${ }^{1}$ Departamento de Ciências do Esporte, Universidade Federal do Triângulo Mineiro (UFTM) - Uberaba (MG), Brasil.

2Programa de Pós-Graduação em Educação Física, Universidade Federal de Santa Catarina (UFSC) - Florianópolis (SC), Brasil.

DOI: http://dx.doi.org/10.7322/abcshs.v41i2.872

\section{RESUMO}

Introdução: A análise da validade de medidas referidas da massa corporal e estatura são fundamentais para aplicação em inquéritos epidemiológicos. Objetivo: Os objetivos deste estudo foram: i) estimar a validade das medidas referidas da massa corporal e estatura, e do índice de massa corporal (IMC) calculado com base nessas medidas; ii) analisar a associação entre as variáveis exploratórias de vínculo com a universidade e sociodemográficas com o erro entre as medidas referidas e aferidas da massa corporal e estatura, e do IMC calculado com base nessas medidas. Métodos: Trata-se de estudo transversal, realizado com estudantes (amostra estimada: 100) de uma universidade pública. As informações referidas da massa corporal e estatura foram obtidas via questionário e a aferição das medidas realizadas em seguida. Foi calculado o excesso de peso corporal $\left(\mathrm{IMC} \geq 25,0 \mathrm{~kg} / \mathrm{m}^{2}\right)$ e os erros entre as medidas referidas e aferidas. As análises incluíram testes para as variáveis em escala quantitativa e qualitativa. Resultados: Participaram deste estudo 146 estudantes e a concordância entre as medidas referidas e aferidas, em escala quantitativa foram satisfatórias. O nível de concordância para o excesso de peso corporal foi forte em homens (kappa=0,76) e mulheres (kappa=0,74). O IMC foi superestimado por homens e mulheres do quartil superior de idade e subestimado pelas estudantes com maior tempo de exposição à universidade. Conclusão: As medidas referidas apresentaram nível satisfatório de validade, e os fatores associados aos erros das medidas referidas foram a maior faixa etária e as mulheres com maior tempo de exposição à universidade.

Palavras-chave: antropometria; índice de massa corporal; validade dos testes; estudantes.

\section{ABSTRACT}

Introduction: The analysis of the validity of the measures referred of body weight and height are essential for use in epidemiological surveys. Objective: The objectives of this study were: i) to estimate the validity of the measures referred of body weight and height, and body mass index (BMI) calculated these measures; ii) to analyze the association between the explanatory variables (link with the university and sociodemographic) with the error between reported and measured body mass and height, and BMI calculated based measures. Methods: It was carried out a cross-sectional study, conducted with students (calculated sample: 100) of a public university. The information referred to body weight and height was obtained through a questionnaire and assessment of the measures undertaken then. It was calculated the excess body weight (BMI: $\geq 25.0 \mathrm{~kg} / \mathrm{m}^{2}$ ) and errors between reported and measured. The analysis included tests for variables in quantitative and qualitative scale. Results: The study included 146 students and the correlation between reported and measured in quantitative scale were satisfactory. The level of agreement for excess body weight was stronger in men (kappa=0.76) and women (kappa=0.74). BMI was overestimated by men and women over the age quartile and underestimated by women with greater exposure time to the university. Conclusion: These measures presented satisfactory level of validity, and the factors associated with errors of the measures mentioned were students of higher age group and women with longer exposure time at the university.

Keywords: anthropometry; body mass index; validity of tests; students. 


\section{INTRODUÇÃO}

A obesidade representa um grave problema de saúde pública nos países desenvolvidos e em desenvolvimento ${ }^{1}$. Para a mensuração dessa morbidade, o índice de massa corporal (IMC), calculado com base nas medidas da massa corporal e estatura tem sido o método mais utilizado ${ }^{1,2}$. A massa corporal e estatura podem ser obtidas pela aferição ou estimadas por meio do relato dos avaliados. Em inquéritos epidemiológicos, principalmente em função do elevado tamanho das amostras, o uso das medidas referidas tem sido empregado ${ }^{1,2}$.

A possibilidade de utilização das medidas referidas, para o cálculo do IMC, é apontada nos estudos de validação em relação às medidas aferidas ${ }^{3-5}$. Em adultos (20 a 59 anos) destacam-se níveis satisfatórios de concordância entre as medidas ${ }^{4,5}$, em contrapartida, para o emprego em estudos com adultos residentes nas áreas rurais $^{6}$, adolescentes ${ }^{3,7}$ e idosos ${ }^{3}$ é necessário cautela.

No Brasil, estudos em relação à validade do uso de medidas referidas da massa corporal e estatura em pesquisas com estudantes universitários ainda são escassos e deste modo um menor conhecimento acerca da utilização dessas medidas nesse grupo populacional, principalmente em função das especificidades sociodemográficas e de vínculo com a universidade. Em pesquisa realizada com estudantes universitários espanhóis, o IMC obtido pelas medidas referidas foi subestimado tanto nos homens quanto nas mulheres, e os níveis de sensibilidade e especificidade para a detecção do excesso de peso corporal foram satisfatórios ${ }^{8}$. Em estudantes universitários tailandeses foi observado que a estatura foi superestimada e a massa corporal subestimada, em ambos os sexos?.

Em virtude do aumento da quantidade de estudantes universitários no Brasil entre 2001 e 2010, especialmente na região nordeste $^{10}$, torna-se necessária a realização de pesquisas com esse grupo para a identificação da prevalência de sobrepeso e a obesidade ${ }^{11,12}$. Considerando a facilidade para o uso das medidas referidas para o calculo do IMC nas pesquisas com grandes amostras nesse grupo, a necessidade da avaliação dos níveis de validade são essenciais. Sendo assim os objetivos deste estudo foram: i) estimar a validade das medidas referidas da massa corporal e estatura, e do IMC calculado com base nessas medidas; ii) analisar a associação entre as variáveis exploratórias de vínculo com a universidade e sociodemográficas com o erro entre as medidas referidas e aferidas da massa corporal e estatura, e do IMC calculado com base nessas medidas.

\section{MÉTODOS}

Esta pesquisa, transversal, faz parte da etapa destinada a testar o uso das medidas referidas da massa corporal e estatura, para fins de cálculo do IMC, utilizados no Estudo MONISA (Monitoramento dos Indicadores de Saúde e Qualidade de Vida de Acadêmicos) ${ }^{13}$. O estudo (a parte dos inquéritos principais) foi realizado em maio de 2014, na instituição onde o Estudo MONISA é desenvolvido.

Para o cálculo amostral considerou-se o erro tipo I de 5\%, erro tipo II de $20 \%$ e correlações de 0,30 , sendo assim a amostra estimada foi de 80 estudantes universitários. Por fim, optou-se por aumentar intencionalmente a amostra para 100 estudantes universitários e estratificar 50 para cada sexo. O cálculo amostral foi realizado no software Statistica, versão 7 .

Os estudantes foram selecionados por conveniência, em diferentes cursos, período de estudo e anos de ingresso na universidade. A coleta dos dados foi realizada em locais estratégicos, com maior fluxo de estudantes, para fins de convite (verbal) para a participação. Os participantes responderam a um questionário sobre as informações de vínculo com a universidade (curso, período de estudo e ano de ingresso na universidade), características sociodemográficas (sexo, idade em anos completos e situação conjugal), massa corporal em quilogramas $(\mathrm{kg})$ e estatura em metros (m). Após o preenchimento das informações, a massa corporal e a estatura foram mensuradas.

Para a realização da coleta de dados, três estudantes do curso de Educação Física foram previamente treinados quanto aos procedimentos de aferição da massa corporal e estatura. Os avaliadores foram testados quanto ao erro técnico de medida (ETM) intra-avaliador e interavaliador das medidas mensuradas, mediante a testagem em 10 estudantes universitários voluntários, do curso de Educação Física, que não fizeram parte da amostra. Os resultados do ETM relativos dos avaliadores deste estudo foram satisfatórios tanto para a massa corporal (Avaliador 1: ETM intra-avaliador, 0\%; ETM interavaliador, 0,7\%; Avaliador 2: ETM intra-avaliador, 0\%; ETM interavaliador, 0,1\%; Avaliador 3: ETM intra-avaliador, 0\%; ETM interavaliador, 0,1\%) quanto para a estatura (Avaliador 1: ETM intra-avaliador, 0\%; ETM interavaliador, 0,9\%; Avaliador 2: ETM intra-avaliador, $0 \%$; ETM interavaliador, 0,6\%; Avaliador 3: ETM intra-avaliador, 0\%; ETM interavaliador, $1 \%)^{14}$.

A medida aferida da massa corporal foi obtida com o mínimo de roupas possíveis, e solicitada à retirada de qualquer objeto que pudesse influenciar na aferição, como por exemplo, sapatos, chaves, relógios e outros. Foi utilizada uma balança digital, da marca Tanita, modelo UM-080, com capacidade máxima de $150 \mathrm{~kg}$ e precisão de 100 gramas. A estatura foi mensurada em uma trena, com fita métrica, metálica e inextensível, da marca Megaforth, com limite máximo de $3 \mathrm{~m}$, precisão de 0,1 centímetros, fixada em parede plana, sem rodapé, e o valor verificado com o auxilio de um nível, equipado com estabilizador em mercúrio. Os procedimentos de aferição seguiram a padronização de Lohman, Roche e Martorell ${ }^{15}$. A estatura foi aferida com os estudantes universitários sem sapatos, em posição ortostática e a cabeça orientada conforme o plano de Frankfurt.

As variáveis dependentes deste estudo foram: massa corporal, estatura e IMC. O IMC foi calculado tendo como base a equação padrão (massa corporal dividido pela estatura ao quadrado) e juntamente com as medidas da massa corporal e estatura foram analisados de forma quantitativa. Além disso, o IMC foi categorizado, considerando os critérios propostos pela Organização Mundial de Saúde ${ }^{16}$ para adultos, com 18 anos ou mais, e definido como excesso de peso corporal o IMC de $25,0 \mathrm{~kg} / \mathrm{m}^{2}$ ou mais. Os estudantes com idade inferior a 18 anos foram classificados conforme Cole et al. ${ }^{17}$. 
As variáveis exploratórias devínculo com a universidade foram: curso (Educação Física e outros cursos, sendo: Administração, Ciências Biológicas, Biomedicina, Ciências Sociais, Ciências da Computação, Comunicação Social, Ciências Econômicas, Engenharia Civil, Engenharia Elétrica, Engenharia Mecânica, Engenharia de Produção, Enfermagem, Filosofia, História, Línguas Estrangeiras Aplicadas às Negociações Internacionais, Letras, Pedagogia e Química); período de estudo (diurno e noturno); anos de exposição à universidade em, $1^{\circ}$ ano (ingresso em 2014), $2^{\circ}$ ano (ingresso em 2013), $3^{\circ}$ ano (ingresso em 2012) e $4^{\circ}$ ano e mais (ingresso em 2011 ou anos anteriores). As variáveis sociodemográficas foram: sexo, idade em quartos (17 a 19 anos, 20 e 21 anos, 22 a 24 anos e 25 a 46 anos) e situação conjugal (sem companheiro e com companheiro).

Os dados foram tabulados em planilha do Excel, versão 2007 e as análises foram realizadas no software STATA, versão 10. Para analisar a validade concorrente foram empregados os seguintes procedimentos:

1) para as variáveis quantitativas (massa corporal, estatura e IMC) foi utilizado o gráfico de dispersão de Bland-Altman ${ }^{18}$, complementadas pelo Coeficiente de Correlação Intraclasse (CCI), tendo em vista a distribuição normal dos dados;

2) para estimar a concordância do excesso de peso corporal foi empregado o teste Kappa (k), e adotada a seguinte classificação: $\leq 0,20=$ concordância leve, pobre; 0,21 a $0,40=$ concordância fraca, regular; 0,41 a $0,60=$ concordância moderada; 0,61 a $0,80=$ concordância forte; $\geq 0,80=$ concordância perfeita $^{19}$, juntamente com as análises de sensibilidade, especificidade, valor preditivo positivo (VPP) e valor preditivo negativo (VPN).

A comparação entre as médias das medidas aferidas e referidas foram realizadas pelo teste $t$ de Student pareado. As prevalências de excesso de peso corporal, tendo como base o IMC calculado pelas medidas aferidas e o IMC calculado pelas medidas referidas, foram comparadas pelo teste McNemar.

Foram calculadas as diferenças entre as variáveis quantitativas (massa corporal, estatura e IMC), das medidas referidas em relação às medidas aferidas, e estimada a associação entre as variáveis exploratórias de vínculo com a universidade e sociodemográficas com essas diferenças. Para tanto, foi empregada a análise de regressão linear múltipla, com o ajuste realizado para todas as variáveis exploratórias de forma simultânea.

As análises descritivas das médias $( \pm)$, frequências absolutas e relativas foram empregadas para a descrição das informações. As análises foram realizadas para todos os estudantes e separadas por sexo. O nível de significância adotado foi de 5\%. Esta etapa do estudo foi aprovada no comitê de ética em pesquisa local e os estudantes que participaram, de forma voluntária, assinaram o termo de consentimento livre e esclarecido.

\section{RESULTADOS}

Da amostra inicialmente calculada houve a participação de 46 estudantes a mais, perfazendo 146 estudantes universitários. A média etária para os homens foi de 22,8 anos $( \pm 5,0)$ e para as mulheres de 21,8 anos $( \pm 5,2)$. Houve maioria de homens $(52,7 \%)$, estudantes sem companheiro $(87,7 \%)$, do período de estudo diurno $(93,8 \%)$, e predomínio de estudantes de 17 a 19 anos (31,0\%) e do $2^{\text {a }}$ ano de exposição à universidade $(38,4 \%)$. Participaram 40,4\% de estudantes do curso de Educação Física.

A Tabela 1 apresenta as informações descritivas das medidas referidas e aferidas. A estatura média referida pelas mulheres

Tabela 1: Análise descritiva e de concordância entre as medidas referidas e aferidas da massa corporal e estatura, e índice de massa corporal mensurado

\begin{tabular}{|c|c|c|c|c|c|c|c|c|c|}
\hline \multirow{2}{*}{ Variáveis } & \multicolumn{3}{|c|}{ Homens } & \multicolumn{3}{|c|}{ Mulheres } & \multicolumn{3}{|c|}{ Total } \\
\hline & $\mathrm{n}$ & Média $\pm D P$ & $\mathrm{CCl}$ & n & Média $\pm D P$ & $\mathrm{CCl}$ & $\mathrm{n}$ & Média $\pm \mathrm{DP}$ & $\mathrm{CCl}$ \\
\hline \multicolumn{10}{|l|}{$\mathrm{MC}(\mathrm{kg})$} \\
\hline Referida & 76 & $71,30( \pm 12,90)$ & \multirow{2}{*}{0,98} & 68 & $59,30( \pm 9,40)$ & \multirow{2}{*}{0,98} & 144 & $65,70( \pm 12,80)$ & \multirow[t]{2}{*}{0,99} \\
\hline Aferida & 76 & $71,4( \pm 12,80)$ & & 69 & $59,00( \pm 9,90)$ & & 145 & $65,50( \pm 13,00)$ & \\
\hline \multicolumn{10}{|l|}{ Estatura $(\mathrm{m})$} \\
\hline Referida & 75 & $1,76( \pm 0,08)$ & \multirow{2}{*}{0,95} & 67 & $1,63( \pm 0,07)^{*}$ & \multirow{2}{*}{0,87} & 142 & $1,70( \pm 0,10)^{\star}$ & \multirow[t]{2}{*}{0,95} \\
\hline Aferida & 76 & $1,75( \pm 0,07)$ & & 69 & $1,62( \pm 0,06)$ & & 145 & $1,69( \pm 0,09)$ & \\
\hline \multicolumn{10}{|l|}{ IMC $\left(\mathrm{kg} / \mathrm{m}^{2}\right)$} \\
\hline Referido & 75 & $23,10( \pm 3,40)$ & \multirow{2}{*}{0,95} & 66 & $22,30( \pm 3,30)$ & \multirow{2}{*}{0,93} & 141 & $22,70( \pm 3,40)$ & \multirow[t]{2}{*}{0,94} \\
\hline \multirow[t]{2}{*}{ Aferido } & 76 & $23,10( \pm 3,40)$ & & 69 & $22,40( \pm 3,40)$ & & 145 & $22,80( \pm 3,40)$ & \\
\hline & $\mathbf{n}$ & $\%$ & k & $\mathbf{n}$ & $\%$ & k & $\mathbf{n}$ & $\%$ & k \\
\hline EPC & 74 & & \multirow{3}{*}{0,76} & 66 & & \multirow{3}{*}{0,74} & 140 & & \multirow{3}{*}{0,76} \\
\hline Referido & & $28,0^{\ddagger}$ & & & $13,6^{\ddagger}$ & & & $21,3^{\ddagger}$ & \\
\hline Aferido & & 28,9 & & & 14,5 & & & 22,1 & \\
\hline Sensibilidade & & 81,0 & & & 77,8 & & & 80,0 & \\
\hline Especificidade & & 94,3 & & & 96,5 & & & 95,5 & \\
\hline VPP & & 85,0 & & & 77,8 & & & 82,8 & \\
\hline VPN & & 92,6 & & & 96,5 & & & 94,6 & \\
\hline
\end{tabular}

DP: desvio padrão; CCl: Coeficiente de Correlação Intraclasse; k: Kappa; MC: Massa corporal; IMC: Índice de massa corporal; EPC: Excesso de peso corporal; VPP: Valor preditivo positivo; VPN: Valor preditivo negativo; ${ }^{\mathrm{p}} \mathrm{p}$ valor $<0,05$ no teste $T$ de Student pareado; ${ }^{\star}$ valor $\mathrm{p}>0,05$ no teste McNemar. 
foi maior que a média aferida, assim como quando considerando todos os universitários. Foi observado entre as medidas referidas e aferidas, correlações positivas e maiores que 0,80 e concordância forte na análise para todos os estudantes e para os homens na estimativa do excesso de peso corporal, além disso, a sensibilidade para a estimativa do excesso de peso corporal, mediante as medidas referidas foi predominante em homens, porém, entre as mulheres foi a especificidade. As prevalências de excesso de peso corporal, estimadas pelo IMC, calculado pelas medidas referidas e pelas medidas aferidas não apresentaram diferenças.

A Figura 1 apresenta a concordância entre as medidas referidas com as aferidas da massa corporal, estatura, e IMC, respectivamente, para todos os estudantes universitários e em separado para o sexo. Observou-se concordância satisfatória entre as medidas. A massa corporal referida foi superestimada em mulheres e a estatura referida foi superestimada por homens e mulheres, mas, principalmente em mulheres. O IMC estimado pelas medidas referidas foi subestimado em homens e em mulheres.

A Tabela 2 (homens) e Tabela 3 (mulheres) apresentam a associação entre as variáveis sociodemográficas e de vínculo com a universidade com as diferenças entre as medidas referidas versus aferidas. Os homens da maior faixa etária subestimaram a estatura em $2 \%$ e superestimaram o IMC que os mais jovens, independente de outras características.

Para as mulheres, a estatura referida foi subestimada pelas estudantes da maior faixa etária, e aquelas do segundo ano e quarto ano e mais de exposição à universidade superestimaram as medidas referidas em 4\%. Por consequência, o IMC das mulheres da maior faixa etária foi superestimado em 1,5 vezes mais que as mais jovens, e as estudantes do $2^{\circ}$ ano e $4^{\circ}$ ano e mais de exposição à universidade subestimaram o IMC.

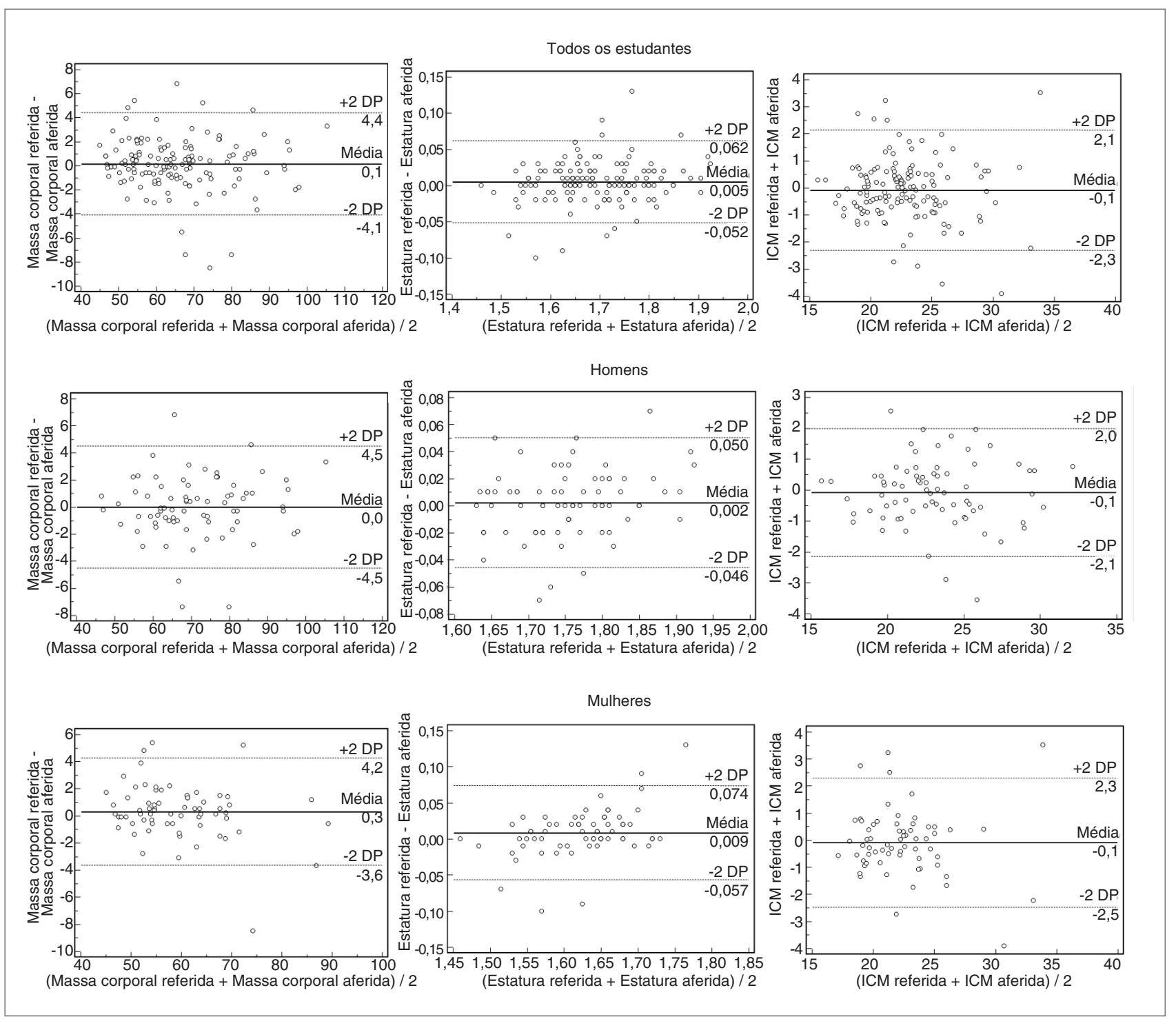

Figura 1: Dispersão de Bland-Altman das medidas da massa corporal, estatura e índice de massa corporal 


\section{DISCUSSÃO}

Este estudo mostrou correlações fortes e positivas, além de concordância satisfatória entre as medidas referidas e aferidas da massa corporal, estatura e IMC em estudantes universitários. A massa corporal referida foi superestimada em mulheres, e a estatura referida foi superestimada tanto em homens quanto em mulheres. O IMC por meio das medidas referidas foi subestimado em homens e em mulheres. Os homens do estrato de maior idade subestimaram a estatura e dessa forma o IMC foi superestimado. As mulheres de maior faixa etária subestimaram a estatura, e aquelas que estavam no $2^{\circ}$ e $4^{\circ}$ ano e mais de exposição à universidade superestimaram a estatura; por consequência o IMC referido foi superestimado em mulheres de maior faixa etária e subestimado por aquelas do $2^{\circ}$ e $4^{\circ}$ ano e mais de exposição à universidade. A concordância para o excesso de peso corporal foi forte e com maior especificidade.

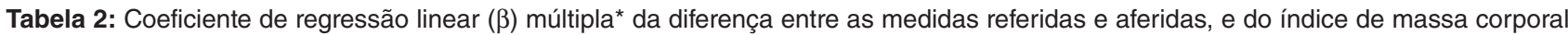
calculado com base nessas medidas em homens

\begin{tabular}{|c|c|c|c|c|c|c|}
\hline \multirow{2}{*}{ Variáveis } & \multicolumn{2}{|c|}{ Massa corporal ${ }^{\ddagger}$} & \multicolumn{2}{|l|}{ Estatura $^{\dagger}$} & \multicolumn{2}{|l|}{$\mathrm{IMC}^{+}$} \\
\hline & $\beta(\mathrm{IC95 \% )}$ & Valor $p$ & $\beta(I C 95 \%)$ & Valor $p$ & $\beta(\mathrm{IC95 \% )}$ & Valor $p$ \\
\hline Faixa etária em terços & & 0,57 & & 0,02 & & \\
\hline 17 a 19 anos & 1,00 & & 1,00 & & 1,00 & \\
\hline 20 e 21 anos & $0,80(-0,92-2,52)$ & & $-0,01(-0,03-0,01)$ & & $0,52(-0,29-1,33)$ & 0,06 \\
\hline 22 a 24 anos & $1,40(-0,42-3,22)$ & & $-0,02(-0,04-0,003)$ & & $0,94(0,08-1,80)$ & \\
\hline 25 a 46 anos & $0,74(-1,04-2,52)$ & & $-0,02(-0,04--0,002)$ & & $0,87(0,03-1,71)$ & \\
\hline Situação conjugal & & 0,08 & & 0,34 & & \\
\hline Sem companheiro & 1,00 & & 1,00 & & 1,00 & 0,06 \\
\hline Com companheiro & $-1,89(-4,05-0,26)$ & & $0,01(-0,01-0,04)$ & & $-0,97(-1,98-0,04)$ & \\
\hline Período de estudo & & 0,36 & & 0,66 & & \\
\hline Diurno & 1,00 & & 1,00 & & 1,00 & 0,68 \\
\hline Noturno & $1,28(-1,52-4,08)$ & & $0,007(-0,02-0,04)$ & & $0,27(-1,04-1,58)$ & \\
\hline Anos de exposição à universidade & & 0,57 & & 0,20 & & \\
\hline $1^{\circ}$ ano & 1,00 & & 1,00 & & 1,00 & \\
\hline $2^{\circ}$ ano & $0,81(-0,77-2,39)$ & & $0,01(-0,01-0,03)$ & & $-0,03(-0,78-0,71)$ & 0,17 \\
\hline 3o ano & $0,55(-1,21-2,31)$ & & $-0,0001(-0,02-0,02)$ & & $0,18(-0,65-1,01)$ & \\
\hline $4^{\circ}$ ano e mais & $-0,82(-2,65-1,01)$ & & $0,02(-0,003-0,04)$ & & $-0,79(-1,65-0,08)$ & \\
\hline Cursos & & 0,24 & & 0,35 & & \\
\hline Educação Física & 1,00 & & 1,00 & & 1,00 & 0,78 \\
\hline Outros & $0,63(-0,43-1,69)$ & & $0,01(-0,01-0,02)$ & & $0,07(-0,43-0,57)$ & \\
\hline
\end{tabular}

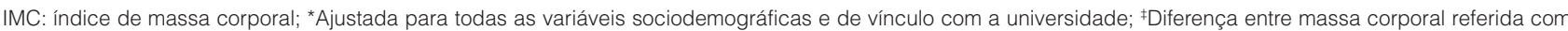
massa corporal aferida; †Diferença entre estatura referida com estatura aferida; + Diferença entre IMC referido com IMC aferido; $\beta$ : coeficiente de regressão linear.

Tabela 3: Coeficiente de regressão linear $(\beta)$ múltipla* da diferença entre as medidas referidas e aferidas, e do índice de massa corporal calculado com base nessas medidas em mulheres

\begin{tabular}{|c|c|c|c|c|c|c|}
\hline \multirow{2}{*}{ Variáveis } & \multicolumn{2}{|c|}{ Massa corporal ${ }^{\ddagger}$} & \multicolumn{2}{|l|}{ Estatura $^{\dagger}$} & \multicolumn{2}{|l|}{$\mathrm{IMC}^{+}$} \\
\hline & $\beta(\mathrm{IC} 95 \%)$ & Valor $p$ & $\beta(\mathrm{IC} 95 \%)$ & Valor $\mathrm{p}$ & $\beta(I C 95 \%)$ & Valor $p$ \\
\hline Faixa etária em terços & & 0,34 & & 0,01 & & \\
\hline 17 a 19 anos & 1,00 & & 1,00 & & 1,00 & \\
\hline 20 e 21 anos & $-1,04(-2,39-0,32)$ & & $-0,01(-0,03-0,006)$ & & $-0,01(-0,76-0,75)$ & 0,01 \\
\hline 22 a 24 anos & $0,37(-1,42-2,17)$ & & $-0,02(-0,05-0,009)$ & & $0,89(-0,13-1,90)$ & \\
\hline 25 a 46 anos & $0,73(-1,42-2,89)$ & & $-0,03(-0,07--0,003)$ & & $1,51(0,31-2,71)$ & \\
\hline Situação conjugal & & 0,18 & & 0,32 & & \\
\hline Sem companheiro & 1,00 & & 1,00 & & 1,00 & 0,79 \\
\hline Com companheiro & $1,21(-0,58-2,99)$ & & $0,01(-0,01-0,04)$ & & $-0,14(-1,14-0,87)$ & \\
\hline Período de estudo & & 0,41 & & 0,35 & & \\
\hline Diurno & 1,00 & & 1,00 & & 1,00 & 0,40 \\
\hline Noturno & $-0,74(-2,53-1,04)$ & & $-0,01(-0,04-0,01)$ & & $0,45(-0,62-1,52)$ & \\
\hline Anos de exposição à universidade & & 0,17 & & 0,02 & & \\
\hline $1^{\circ}$ ano & 1,00 & & 1,00 & & 1,00 & \\
\hline $2^{\circ}$ ano & $-0,23(-1,76-1,30)$ & & $0,04(0,01-0,06)$ & & $-1,15(-2,00--0,31)$ & 0,01 \\
\hline $3^{\circ}$ ano & $0,41(-1,45-2,27)$ & & $0,03(-0,001-0,05)$ & & $-0,74(-1,76-0,28)$ & \\
\hline $4^{\circ}$ ano e mais & $-0,79(-2,74-1,16)$ & & $0,04(0,01-0,07)$ & & $-1,80(-2,88--0,72)$ & \\
\hline Cursos & & 0,67 & & 0,18 & & \\
\hline Educação Física & 1,00 & & 1,00 & & 1,00 & 0,56 \\
\hline Outros & $0,23(-0,83-1,29)$ & & $0,01(-0,005-0,03)$ & & $-0,18(-0,78-0,42)$ & \\
\hline
\end{tabular}

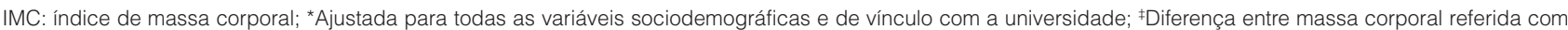
massa corporal aferida; †Diferença entre estatura referida com estatura aferida; +Diferença entre IMC referido com IMC aferido; $\beta$ : coeficiente de regressão linear. 
Correlações positivas e elevadas entre as medidas referidas e aferidas da massa corporal, estatura e IMC é condizente com outros autores ${ }^{8,9}$. Estudos de validação dessas medidas em estudantes universitários espanhóis ${ }^{8}$ e tailandeses ${ }^{9}$ mostraram correlações positivas e maiores que 0,93 , tanto em homens quanto em mulheres.

As pessoas na fase adulta tendem a apresentar menores vieses de resposta ${ }^{3}$, além disso, maior nível de escolaridade contribui com maior validade para o uso dessas medidas. No Brasil, o período universitário compreende a faixa de 18 a 24 anos, especialmente nas regiões sul, sudeste e centro-oeste. Nas regiões norte e nordeste o perfil é caracterizado por estudantes universitários com idades mais avançadas, porém, inferiores a 60 anos, dessa forma sendo possível a utilização do autorrelato das medidas da massa corporal e estatura ${ }^{20}$.

No presente estudo, as mulheres, superestimaram a massa corporal referida $(0,3 \mathrm{~kg})$ e a estatura referida foi superestimada por homens e mulheres, $\sim 0,01 \mathrm{~cm}$, similar ao verificado em outros estudos ${ }^{8,9}$. Em pesquisa realizada com estudantes universitários espanhóis, as mulheres superestimaram a massa corporal referida, em $0,02 \mathrm{~kg}$, e os homens e as mulheres superestimaram a estatura, $0,24 \mathrm{e} 0,28 \mathrm{~cm}$, respectivamente ${ }^{8}$. Estudantes universitários tailandeses também superestimaram a estatura (1,5 $\mathrm{cm}$ os homens e $1,3 \mathrm{~cm}$ as mulheres) ${ }^{9}$.

O IMC obtido por meio das medidas referidas foi subestimado em homens e em mulheres, assim como verificado em pesquisa com estudantes universitários espanhóis ${ }^{8}$ e tailandeses ${ }^{9}$. Em outros estudos de validação realizados com adolescentes, o IMC calculado com as informações referidas é divergente, com a subestimação em adolescentes de 15 a 19 anos $^{21}$ ou a superestimação dos valores para aqueles com idades de 10 a 14 anos $^{7}$. Em adultos brasileiros ${ }^{22}$ e portugueses ${ }^{23}$, os resultados foram semelhantes aos deste estudo, com os resultados do IMC tendo como base as informações referidas subestimadas.

Tanto para os homens quanto para as mulheres da faixa etária de 25 a 46 anos houve pouca subestimativa da estatura, em 0,02 e $0,03 \mathrm{~cm}$, respectivamente, e por isso o IMC foi superestimado. Os resultados deste estudo, em relação à associação entre a idade e a estatura referida foram semelhantes ao observado em universitários espanhóis ${ }^{8}$. Importante destacar que nesse estudo o limite superior de idade foi 46 anos, e o uso dessas medidas em pessoas com idade acima de 60 anos deve ser realizado com cautela ${ }^{3}$.

Dentre as variáveis de vínculo com a universidade, apenas as mulheres, que estavam no $2^{\circ} \mathrm{e} 4^{\circ}$ ano de exposição à universidade superestimaram a estatura e o IMC foi subestimado, independente das outras variáveis. Possíveis explicações são complexas em relação à associação do tempo de exposição universitária sobre a diferença entre a medida mensurada por autorrelato e a aferida, contudo, mulheres com maior escolaridade superestimam a estatura ${ }^{22}$.

Em relação à concordância entre as medidas, os resultados mostraram que os valores para o excesso de peso corporal foram satisfatórios, tanto em homens quanto em mulheres. Houve menor sensibilidade do que especificidade para os sexos, de forma predominante para as mulheres. Em estudantes universitários espanhóis, a concordância referente aqueles com ou sem excesso de peso corporal (sobrepeso mais obesidade), foi em mulheres de $\mathrm{k}=0,80$, e em homens $\mathrm{k}=0,63^{8}$, o que difere deste estudo. No presente estudo, o nível de concordância foi semelhante ao verificado em estudos com adultos de diferentes níveis de escolaridade ${ }^{22,24} \mathrm{e}$ com adolescentes de 14 a 19 anos $^{25}$.

As limitações do estudo estão relacionadas a não calibração do uso da balança digital para a mensuração da massa corporal, que pode estimar com erro a massa corporal, no entanto, esse tipo de equipamento apresenta uma boa aplicabilidade para estudos de validação ${ }^{25}$; o procedimento de seleção adotado neste estudo pode ser caracterizado como uma limitação (seleção por conveniência), pois, possivelmente pode inibir a participação de estudantes universitários com obesidade, bem como favorecer a participação de universitários de cursos específicos, conforme foi verificado neste estudo, com a participação de universitários do curso de Educação Física ${ }^{22,24}$.

Os níveis de concordância das medidas referidas da massa corporal e estatura foram satisfatórios e caracterizam a boa aplicabilidade em estudantes universitários, e para a estimativa do excesso de peso corporal. Os fatores associados ao erro entre as medidas referidas e aferidas foram os estudantes de maior faixa etária, e entre as mulheres com maior tempo de exposição universitária.

\section{REFERÊNCIAS}

1. Low S, Chin MC, Deurenberg-Yap M. Review on epidemic of obesity. Ann Acad Med Singapore. 2009;38(1):57-65.

2. Araújo VC, Konrad LM, Rabacow FM, Graup S, Amboni R, Farias Junior JC. Prevalência de excesso de peso em adolescentes brasileiros: um estudo de revisão sistemática. Rev Bras Ativ Fís Saúde. 2012;12(3):79-87.

3. Coqueiro RS, Borges LJ, Araújo VC, Pelegrini A, Barbosa AR. Medidas auto-referidas são válidas para avaliação do estado nutricional na população brasileira? Rev Bras Cineantropom Desempenho Hum. 2009;11(1):113-19

4. Fonseca MJM, Faerstein E, Chor D, Lopes CS. Validade de peso e estatura informados e índice de massa corporal: estudo prósaúde. Rev Saúde Pública. 2004;38(3):392-8.
5. Merrill RM, Richardson JS. Validity of self-reported height, weight, and body mass index: findings from the National Health and Nutrition Examination Survey, 2001-2006. Prev Chronic Dis. 2009;6(4):A121.

6. Martins PC, Carvalho MB, Machado CJ. Uso de medidas autorreferidas de peso, altura e índice de massa corporal em uma população rural do nordeste brasileiro. Rev Bras Epidemiol. 2015;18(1):137-48. http://dx.doi.org/10.1590/1980-5497201500010011

7. Enes CC, Pegolo GE, Silva MV. Medidas autorreferidas versus medidas aferidas de peso e altura de adolescentes residentes em áreas rurais de Piedade, São Paulo. Nutrire. 2009;34(2):59-70. 
8. Savane FR, Navarrete-Muñoz EM, Hera MG, Gimenez-Monzo D, Gonzalez-Palacios S, Valera-Gran D, et al. Validez del peso y talla auto-referido en población universitaria y factores asociados a las discrepancias entre valores declarados y medidos. Nutr Hosp. 2013;28(5):1633-8. http://dx.doi.org/10.3305/nh.2013.28.5.6671

9. Lim LL, Seubsman S, Sleigh A. Validity of self-reported weight, height, and body mass index among university students in Thailand: Implications for population studies of obesity in developing countries. Popul Health Metr. 2009;7(9):15

http://dx.doi.org/10.1186/1478-7954-7-15

10. Instituto Nacional de Estudos e Pesquisas Educacionais Anísio Teixeira (INEP). Censo da educação superior 2010: Divulgação dos principais resultados do Censo da Educação Superior 2010. Disponível em: http://download.inep.gov.br/educacao_superior/ censo_superior/documentos/2010/divulgacao_censo_2010.pdf. Acesso em: 14 jul. 2012.

11. Legnani RFS, Legnani E, Pereira EF, Gasparotto GS, Vieira LF, Campos W. Transtornos alimentares e imagem corporal em acadêmicos de Educação Física. Motriz: Rev Educ Fís. 2012;18(1):84-91.

http://dx.doi.org/10.1590/S1980-65742012000100009

12. Guedes DP, Legnani RFS, Legnani E. Motivos para a prática de exercício físico em universitários de acordo com o índice de massa corporal. Rev Bras Ativ Fís Saúde. 2012;17(4):270-4.

13. Sousa TF, Fonseca SA, José HPM, Nahas MV. Estudo MONISA: características e aspectos metodológicos. Rev Bras Epidemiol. 2012;15(4):904-7. http://dx.doi.org/10.1590/S1415-790X2012000400020

14. Silva DAS, Pelegrini A, Pires-Neto CS, Vieira MFS, Petroski EL. O antropometrista na busca de dados mais confiáveis. Rev Bras Cineantropom Desempenho Hum. 2011;13(1):82-5. http://dx.doi.org/10.5007/1980-0037.2011v13n1p82

15. Lohman TG, Roche AF, Martorell R. Anthropometric Standardization Reference Manual. Champaign: Human Kinetics Books; 1988
16. World Health Organization (WHO). Obesity: preventing and managing the Global Epidemic. Report on a WHO Expert Consultation on Obesity. Geneva: OMS, 1997.

17. Cole TJ, Bellizzi MC, Flegal KM, Dietz WH. Establishing a standard definition for child overweight and obesity worldwide: international survey. BMJ. 2000;320:1240.

http://dx.doi.org/10.1136/bmj.320.7244.1240

18. Bland JM, Altman DG. Statistical methods for assessing agreement between two methods of clinical measurement. Lancet. 1986;327(8476):307-10.

19. Landis JR, Koch GG. The measurement of observer agreement for categorical data. Biometrics. 1977;33(1):159-74. http://dx.doi.org/10.2307/2529310

20. Ministério da Educação. Instituto Nacional de Estudos e Pesquisas Educacionais Anísio Teixeira (INEP). Censo da educação superior 2011: resumo técnico. Brasília: INEP; 2013.

21. Romanzini M, Reichert FF, Ronque ERV, Lopes AS, Petroski EL. Determinação do estado nutricional de adolescentes por meio de medidas referidas de peso e estatura: um estudo de validação. Rev Bras Ativ Fís Saúde. 2011;16(1):31-6.

22. Del Duca GF, González-Chica DA, Santos JV, Knuth AG, Camargo MBJ, Araújo CL. Peso e altura autorreferidos para determinação do estado nutricional de adultos e idosos: validade e implicações em análises de dados. Cad Saúde Pública. 2012;28(1):75-85. http://dx.doi.org/10.1590/S0102-311X2012000100008

23. Santos O, do Carmo I, Camolas J, Vieira J. Validade do auto-relato do peso e da altura na avaliação do índice de massa corporal da população adulta portuguesa. Endocrinol Diab Obe. 2009;3(4):157-68.

24. Silveira EA, Araújo CL, Gigante DP, Barros AJD, Lima MS. Validação do peso e altura referidos para o diagnóstico do estado nutricional em uma população de adultos no Sul do Brasil. Cad Saúde Pública. 2005;21(1):235-45. http://dx.doi.org/10.1590/S0102-311X2005000100026

25. Rodrigues PRM, Gonçalves-Silva RMV, Pereira RA. Validity of selfreported weight and stature in adolescents from Cuiabá, CentralWestern Brazil. Rev Nutr. 2013;26(3):283-90. http://dx.doi.org/10.1590/S1415-52732013000300003 